\title{
Molecular characteristics of a fluorescent chemosensor for the recognition of ferric ion based on photoresponsive azobenzene derivative
}

\author{
Zhen Chi, Xia Ran", Lili Shi, Jie Lou, Yanmin Kuang and Lijun Guo ${ }^{*}$ \\ Institute of Photobiophysics, School of Physics and Electronics, Henan University, Kaifeng, P. R. \\ China \\ Address correspondence to: Xia Ran, Lijun Guo, School of Physics and Electronics, Henan \\ University, Kaifeng 475004, P. R. China. Email: ranxia@henu.edu.cn, juneguo@henu.edu.cn.
}

Abstract: Metal ion recognition is of great significance in biological and environmental detection. So far, there is very few research related to the ferric ion sensing based on photoresponsive azobenzene derivatives. In this work, we report a highly selective fluorescent "turn-off” sensor for $\mathrm{Fe}^{3+}$ ions and the molecular sensing characteristics based on an azobenzene derivative, N-(3,4,5-octanoxyphenyl)-N'-4-[(4-hydroxyphenyl)azophenyl]1,3,4-oxadiazole (AOB-t8). The binding association constant was determined to be $6.07 \times 10^{3} \mathrm{M}^{-1}$ in ethanol and the stoichiometry ratio of 2:2 was obtained from Job's plot and MS spectra. The AOB-t8 might be likely to form the dimer structure through the chelation of ferric ion with the azobenzene moiety. Meanwhile, it was found that the photoisomerization property of $\mathrm{AOB}-\mathrm{t} 8$ was regulated by the binding with $\mathrm{Fe}^{3+}$. With the chelation of $\mathrm{Fe}^{3+}$, the regulated molecular rigidity and the perturbed of electronic state and molecular geometry was suggested to be responsible for the accelerated isomerization of AOB-t8 to UV irradiation and the increased fluorescence lifetime of both trans- and cis-AOB-t8-Fe(III). Moreover, the reversible sensing of AOB-t8 was successfully observed by releasing the iron ion from AOB-t8-Fe(III) with the addition of citric acid.

Keywords: Azobenzene derivatives, Fluorescence sensor, Ferric ion, Photoisomerization

\section{Introduction}

Metal ion recognition is of significance in supramolecular chemistry and plays an important role for the potential applications in environmental, chemical and biological fields, and a variety of fluorescent sensors of metal ion have been reported in the past decades[1-4]. Chemosensors are compounds demonstrating remarkable changes in their electronic, magnetic or optical properties in binding with specific guests, and have attracted increasing interests in various fields due to the 
inherent simplicity, highly sensitive and selective features[5, 6]. In recent years, a large number of works about detection of $\mathrm{Pb}^{2+}, \mathrm{Zn}^{2+}, \mathrm{Cu}^{2+}$, and $\mathrm{Hg}^{2+}$ have been reported by monitoring the fluorescence properties of chemosensors[7-15]. In the fields of life science, iron ion is a highly important metal ion for living organisms and plays a significant role in many biochemical processes. The trivalent form of iron is the most abundant essential inhuman body[16], acting as a cofactor in many enzymatic reactions and providing the oxygen-carrying capacity of proteins. The overloading or deficiency of $\mathrm{Fe}^{3+}$ can induce various disorders with iron trafficking, such as cancer, neurodegenerative and cardiovascular diseases[17, 18]. So far, there have been many fluorescent sensors for $\mathrm{Fe}^{3+}$ reported in recent years[19, 20]. However, the development of fluorescent chemosensor with variable conformation for $\mathrm{Fe}^{3+}$ is still of significant research potential.

Azobenzene and its derivatives are characterized with their photochromic properties because they can undergo photoinduced trans-cis isomerization [21-23]. In the past decades, they have been widely used as photo-responsive materials, such as liquid crystal, holographicsurface relief grating and optical data storage [24-26]. Recently, azobenzene derivatives were presented as a photoswitchs by coupling the photoisomerization moiety to regulate the function/structure of numerous biomolecular targets, including proteins, peptides, and nucleic acids in vitro and vivo[27, 28]. For example, Liang et al reported that DNA triplex formation was regulated by using cis-trans isomerization of azobenzene [29]. Wong et al presented an azobenzene moiety to be used as a multiresponsive end-cap in self-immolative polymer[30]. Samanta and colleagues demonstrated a tetra-ortho-substituted azobenzene compound with the switching properties to red light[31]. However, vanishingly few papers about azobenzene derivatives were reported in the application of fluorescent sensor for transition metals. It would be of considerable significance in developing azobenzene derivatives-based fluorescent sensors for detection ferric irons, especially in environmental and physiological conditions. In this work, we report the sensing characteristics for ferric ion recognition based on an azobenzene derivative (N-(3,4,5-octanoxyphenyl)N'-4-[(4-hydroxyphenyl)azophenyl]1,3,4-oxadiazole) (AOB-t8). The photoresponsive AOB-t8 demonstrates a turn-off sensor with high selectivity toward $\mathrm{Fe}^{3+}$ among 14 metal ions, and possesses a fluorescent "ON-OFF-ON" functional mode via alternately binding $\mathrm{Fe}^{3+}$ and $\mathrm{C}_{6} \mathrm{H}_{5} \mathrm{O}_{7}{ }^{3-}$ along with reversible formation/separation of the complex in ethanol. Meanwhile, the 
isomerization process of AOB-t8 to UV irradiation can be accelerated by coordinating with $\mathrm{Fe}^{3+}$ ion due to the enhanced molecular rigidity or coplanarity.

\section{Experimental section}

\subsection{Materials}

All the chemicals and reagents were obtained from commercial suppliers and used as received. The solutions of the selected metal ions were prepared from $\mathrm{MgCl}_{2} \cdot 6 \mathrm{H}_{2} \mathrm{O}, \mathrm{Co}\left(\mathrm{NO}_{3}\right)_{2} \cdot 6 \mathrm{H}_{2} \mathrm{O}, \mathrm{KOH}$, $\mathrm{NaOH}, \mathrm{Ni}\left(\mathrm{NO}_{3}\right)_{2} \cdot 6 \mathrm{H}_{2} \mathrm{O}, \mathrm{ZnCl}_{2}, \mathrm{CuCl}_{2} \cdot 2 \mathrm{H}_{2} \mathrm{O}, \mathrm{HgCl}_{2}, \mathrm{AgNO}_{3}, \mathrm{FeCl}_{3} \cdot 6 \mathrm{H}_{2} \mathrm{O}, \mathrm{CaCl}_{2}, \mathrm{Al}\left(\mathrm{NO}_{3}\right)_{3} \cdot 9 \mathrm{H}_{2} \mathrm{O}$, $\mathrm{La}\left(\mathrm{NO}_{3}\right)_{3} \cdot 6 \mathrm{H}_{2} \mathrm{O}, \mathrm{Nd}\left(\mathrm{NO}_{3}\right)_{3} \cdot 6 \mathrm{H}_{2} \mathrm{O}$, respectively.

\subsection{Synthesis and Characterization}

The molecular structure of trans-AOB-t8 is shown in Scheme 1. The synthesis of AOB-t8 was followed the procedure reported in our previous work[32]. Fourier transform infrared (FT-IR) spectra were recorded on a Perkin-Elmer spectrometer with $\mathrm{KBr}$ pellets. UV-visible diffuse reflectance spectra were measured using a Perkin-Elmer Lambda 35 spectrometer. Fluorescence spectra in solution were recorded on a Perkin-Elmer LS-55 fluorescence spectrophotometer. The MS spectra were performed on autoflex ${ }^{\mathrm{TM}}$ speed MALDI-TOF-MS (Bruker). The photoluminescence lifetime measurements were carried out on a home-built lifetime setup (Harp300, Picoquant), coupled with the TCSPC module (time resolution $<4 \mathrm{ps)}$ and a broadband pulsed femtosecond laser (Chameleon Ultra II, Coherent Inc.) with the repetition rate of $80 \mathrm{MHz}$ and pulse width of $150 \mathrm{fs}$, respectively. The $355 \mathrm{~nm}$ femtosecond laser was obtained from the secondary harmonic generation with $\mathrm{BBO}$ nonlinear crystal and was used as the excitation source, and the fluorescence $(>430 \mathrm{~nm})$ was detected with a single photon device (Picoquant, time response $<50 \mathrm{ps}$ ). The polarization configuration for the excitation and emission was set at the magic angle $\left(54.7^{\circ}\right)$, and the impulse response function was measured to be around 50 ps. The UV light for photoisomerization was obtained from a $250 \mathrm{~W}$ high-pressure mercury lamp with a 365 nm band-pass filter.

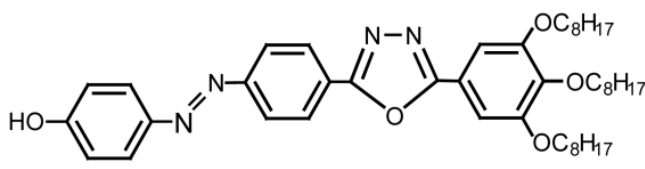

Scheme 1 The molecular structure of trans-AOB-t8. 


\section{Results and discussion}

\subsection{Ion response of trans-AOB-t8}

It is a known fact that azobenzene molecules fluoresce in solution with imperceptible quantum yield. With the molecular grafting of oxadiazole with azobenzene, trans-AOB-t8 exhibits a considerable emission and cis-AOB-t8 demonstrates an enhanced florescence, which can thus be served as the sensing parameter[25]. As shown in Figure 1(a), the UV-visible spectrum of trans-AOB-t8 in ethanol presents an intense absorption at $363 \mathrm{~nm}$, which is attributed to the $\pi-\pi^{*}$ transition. The emission maxima at $433 \mathrm{~nm}$ in the fluorescence spectrum of trans-AOB-t8 can be observed in ethanol with the excitation at $350 \mathrm{~nm}$. In order to investigate the response of trans-AOB-t8 to metal ions, the UV/vis absorption measurements of trans-AOB-t8 $(10 \mu \mathrm{M})$ were performed in the presence of 5 equiv of 14 different metal ions and the results are shown in Figure 1(b). It can be found that in the cases of $\mathrm{K}^{+}, \mathrm{Ni}^{2+}, \mathrm{Mg}^{2+}, \mathrm{Ca}^{2+}, \mathrm{Na}^{+}, \mathrm{Fe}^{3+}, \mathrm{Co}^{2+}, \mathrm{Ag}^{+}, \mathrm{Hg}^{2+}, \mathrm{Zn}^{2+}, \mathrm{Cu}^{2+}, \mathrm{Al}^{3+}$, $\mathrm{La}^{3+}$ and $\mathrm{Nd}^{3+}$, the corresponding absorption spectrum has a very little change. However, the striking difference of absorption from that of free AOB-t8 in ethanol can be observed with the addition of $\mathrm{Fe}^{3+}$. Besides the increase of absorbance, an obvious blue-shift of the $\pi-\pi^{*}$ absorption maximum from $363 \mathrm{~nm}$ to $356 \mathrm{~nm}$ is clearly demonstrated, indicating the perturbation of electronic state and the formation of a new complex with the binding of trans-AOB-t8 with $\mathrm{Fe}^{3+}$ ion [33].
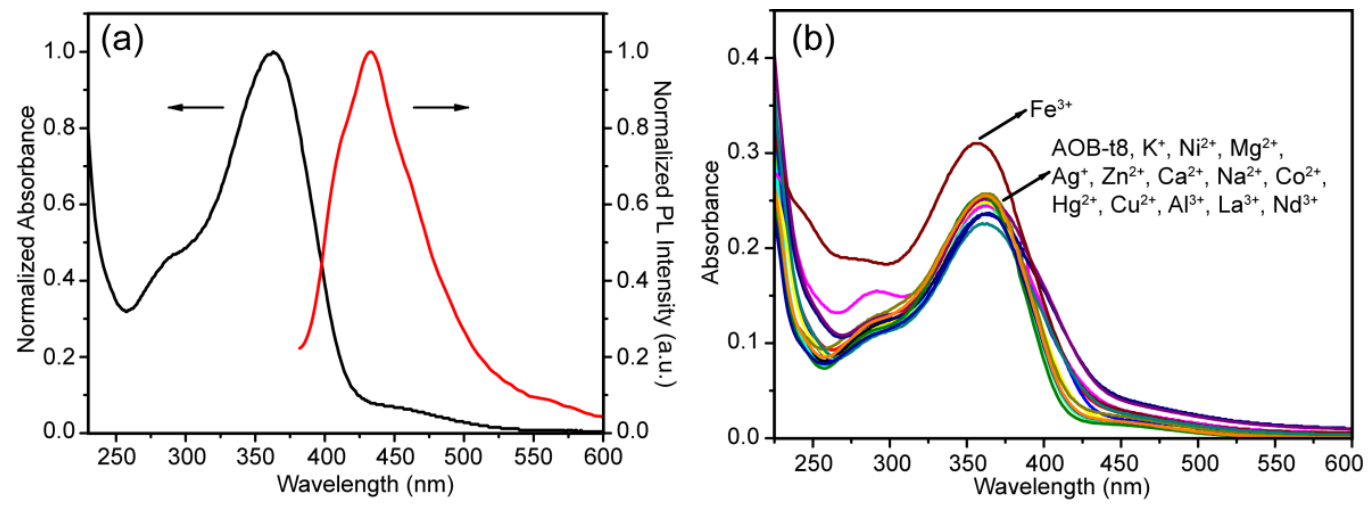

Figure 1 (a) UV-visible and emission spectra of trans-AOB-t8 (10 $\mu \mathrm{M})$ in ethanol, (b) UV-visible spectra of trans-AOB-t8 $(10 \mu \mathrm{M})$ in ethanol upon addition of 14 metal ions (50 $\mu \mathrm{M})$. 
To further explore the characteristics of trans-AOB-t8 as an ion-selective fluorescent sensor, the fluorogenic behavior of trans-AOB-t8 was investigated with the presence of different metal cations. In the experiments, the concentration of trans-AOB-t8 was set as low as $10 \mu \mathrm{M}$ to avoid the aggregation-caused quenching (ACQ) effect. With the addition of 40 equiv of $\mathrm{K}^{+}, \mathrm{Ni}^{2+}, \mathrm{Mg}^{2+}, \mathrm{Ca}^{2+}, \mathrm{Na}^{+}, \mathrm{Fe}^{3+}, \mathrm{Co}^{2+}, \mathrm{Ag}^{+}, \mathrm{Hg}^{2+}, \mathrm{Zn}^{2+}, \mathrm{Cu}^{2+}, \mathrm{Al}^{3+}, \mathrm{La}^{3+}$ and $\mathrm{Nd}^{3+}$, the recorded fluorescence spectra are shown in Figure 2a. It can be found that the presence of $\mathrm{Fe}^{3+}$ leads to the dramatically quenched fluorescence of trans-AOB-t8, while the other test metal ions cause the relatively insignificant change in fluorescence. As a consequence, the color change of trans-AOB-t8 solution induced by ferric ion over other metal ions is distinguishable under the illumination of UV lamp (Figure S1).The highly selective recognition for ferric ion indicates an effective binding of AOB-t8 with $\mathrm{Fe}^{3+}$, probably through electron transfer or energy transfer. To further confirm the selectivity for $\mathrm{Fe}^{3+}$ ion over other metal ions, the metal ion competition experiments were carried out and the results are shown in Figure $2 \mathrm{~b}$. It can be observed that there is nearly no interference for the detection of $\mathrm{Fe}^{3+}$ from all other metal ions.
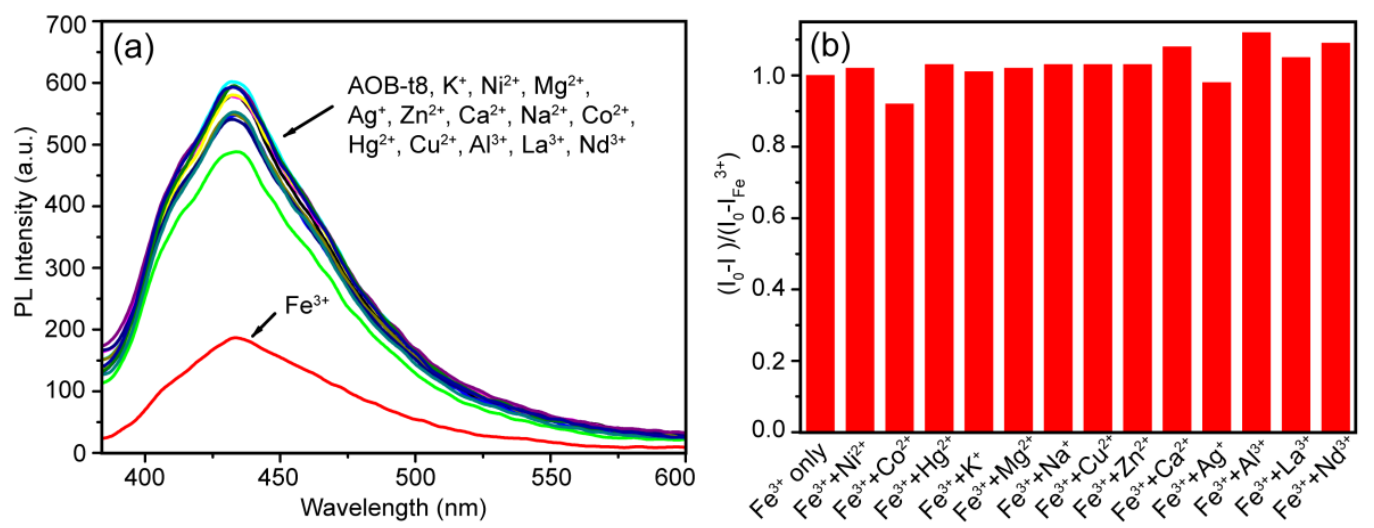

Figure 2 (a) Fluorescence emission spectra of trans-AOB-t8 in ethanol upon addition of different metal ions, (b) Competitive experiments of AOB-t8-Fe(III) system with interfering metal ions. $[\mathrm{AOB}-\mathrm{t} 8]=10 \mu \mathrm{M}$, and $\left[\mathrm{M}^{\mathrm{n}+}\right]=40 \times 10^{-5} \mathrm{M}$, with the excitation at $350 \mathrm{~nm}$ and emission collected at $433 \mathrm{~nm}$.

In order to gain more insight into the sensor property of trans-AOB-t8 toward $\mathrm{Fe}^{3+}$ ion, the response dependence on the concentration of $\mathrm{Fe}^{3+}$ ion is shown in Figure 3. As expected, the fluorescence intensity decreases with the increasing concentration of $\mathrm{Fe}^{3+}$ ion. The $50 \%\left(\left(I_{0}-I\right) / I_{0} \times 100 \%\right)$ of total intensity at the emission maximum can be obtained 
with the 20 equiv of $\mathrm{Fe}^{3+}$ ion, where $I$ and $I_{0}$ represent the fluorescence intensity with and without ferric ion, respectively. On the basis of Stern-Volmer analysis (Figure S2), the quenching effect can be described with $\left(I_{0^{-}} I\right) / I$ versus $\left[\mathrm{Fe}^{3+}\right]$, where $I_{0}$ and $I$ are the fluorescence intensities in the absence and presence of ferric ion. It can be seen that the Stern-Volmer plot over the $\mathrm{Fe}^{3+}$ ions concentration ranging from 0 to $9 \times 10^{-4} \mathrm{M}$ demonstrates a linear nature, indicating a static mechanism for the charge transfer between AOB-t8 and ferric ion. The calculated $K_{\mathrm{SV}}$ is of $6.07 \times 10^{3} \mathrm{M}^{-1}$ for $\mathrm{Fe}^{3+}$ ion, mainly corresponding to the association constant $K_{\mathrm{S}}$ for the current sensor $[34,35]$.To determine the detection limit, the relative fluorescence intensity at $433 \mathrm{~nm}$ was plotted as a function of $\mathrm{Fe}^{3+}$ concentration (Figure S3), the detection limit based on the definition IUPAC $(\mathrm{CDL}=3 \mathrm{Sb} / \mathrm{m})[36]$ is found to be $16.8 \mu \mathrm{M}$ from 8 blank solutions.

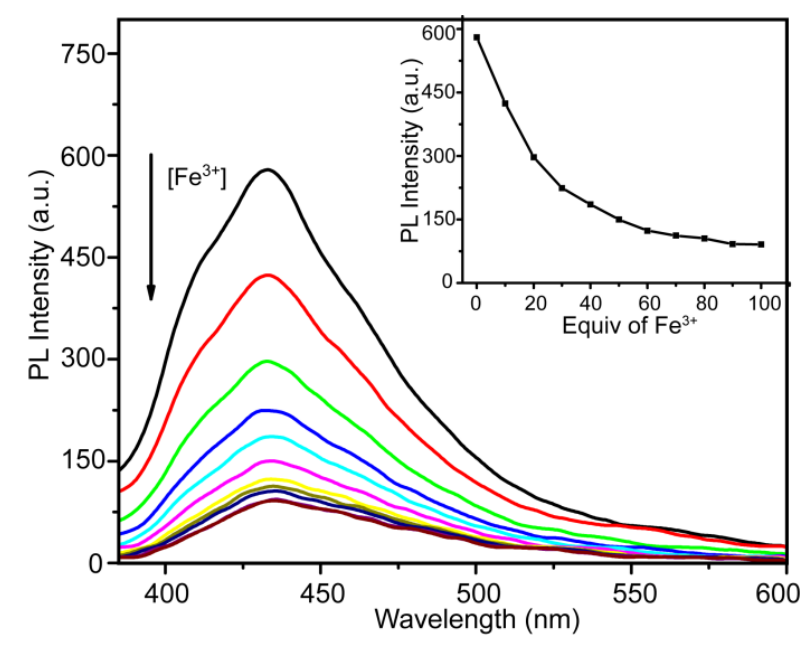

Figure 3 Fluorescence spectra of trans-AOB-t 8 with different concentrations of $\mathrm{Fe}^{3+}(0,1$, $2,3,4,5,6,7,8,9,10) \times 10^{-4} \mathrm{M}$ in ethanol. The excitation wavelength was $350 \mathrm{~nm}$, [AOB-t8] $=10 \mu \mathrm{M}$. Inset shows the fluorescence intensity at $433 \mathrm{~nm}$ as a function of $\mathrm{Fe}^{3+}$ ions.

\subsection{Binding mode of $A O B-t 8$ with $\mathrm{Fe}^{3+}$}

In the conjugated AOB-t8, there are several possible sites to chelate with ferric ion, such as $-\mathrm{OH}, \mathrm{N}=\mathrm{N}$ and $\mathrm{N}-\mathrm{N}$, which can lead to the fluorescence quenching. To find out the molecular configuration of AOB-t8-Fe(III) complex, the binding stoichiometry experiments were carried out with a constant concentration of $\mathrm{AOB}-\mathrm{t} 8$ and $\mathrm{Fe}^{3+}$. As shown in Figure 4, the emission intensity at $433 \mathrm{~nm}$ is plotted against the molarfraction of $\mathrm{Fe}^{3+}$. 
From the obtained Job's plot, an inflection point at 0.5 molar fraction of $\mathrm{Fe}^{3+}$ can be clearly observed, indicating a $1: 1$ or $2: 2$ ratio between $\mathrm{AOB}-\mathrm{t} 8$ and $\mathrm{Fe}^{3+}$ in the inclusion complex[37].

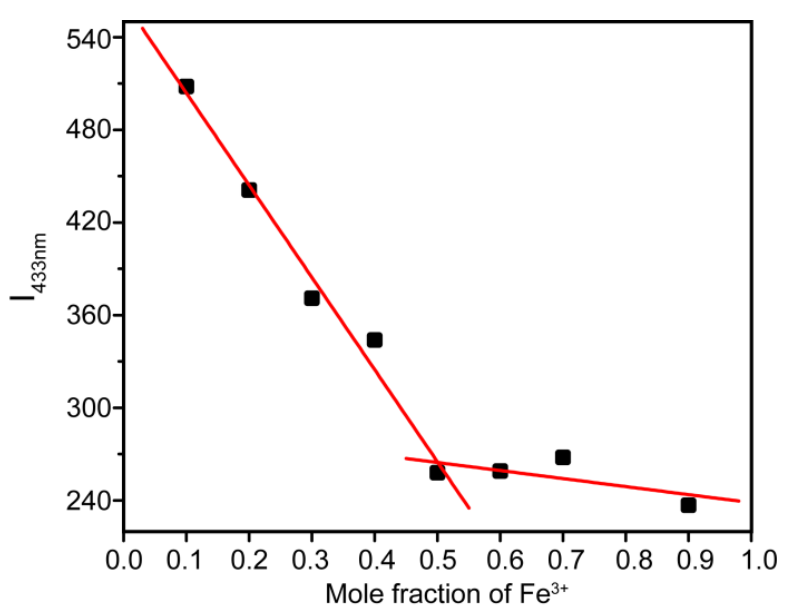

Figure 4 Job's plot of compound AOB-t8 with $\mathrm{Fe}^{3+}$ according to the method of continuous variation. The total concentration of $\mathrm{AOB}-\mathrm{t} 8$ and $\mathrm{Fe}^{3+}$ in ethanol is $10 \mu \mathrm{M}$.

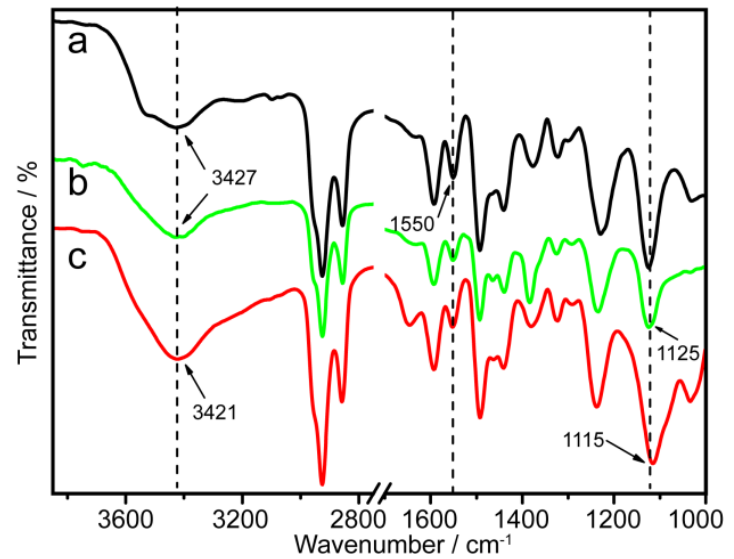

Figure 5 FT-IR spectra of (a) free AOB-t8, (b) AOB-t8@ $\mathrm{Co}^{2+}$, and (c) AOB-t8@ $\mathrm{Fe}^{3+}$.

Vibrational spectroscopy can provide information on the coordination bond of metal ions and sensors. To investigate the binding site of AOB-t8 with the metal ions, FTIR spectroscopies of solid AOB-t8 and AOB-t8@ $\mathrm{Co}^{2+}$ and AOB-t8@ $\mathrm{Fe}^{3+}$ were performed. The spectral data in Figure 5a indicate that the O-H and C-N group stretching vibration modes of AOB-t8 are located at 3427 and $1125 \mathrm{~cm}^{-1}$, respectively[32]. The peak at 1550 $\mathrm{cm}^{-1}$ is attributed to the $\mathrm{C}=\mathrm{N}$ stretching vibration of 1,3,4-oxadiazole ring[38]. After adding $\mathrm{Fe}^{3+}$, the peak of $\mathrm{O}-\mathrm{H}$ vibrational mode is slightly shifted to lower wavenumber of $3421 \mathrm{~cm}^{-1}$ and the stretching vibration peak of C-N appears at $1115 \mathrm{~cm}^{-1}$ (Figure 5c). However, the $\mathrm{C}=\mathrm{N}$ stretching vibration has no change before and after adding $\mathrm{Fe}^{3+}$, 
indicating that $\mathrm{Fe}^{3+}$ is not binding with $\mathrm{C}=\mathrm{N}$ group in oxadiazole moiety. Furthermore, there is almost no observable change in the FTIR of AOB-t8@ $\mathrm{Co}^{2+}$ (Figure 5b) as well, consisting with the ion response data in Figure $1 \mathrm{~b}$, due to no binding between $\mathrm{Co}^{2+}$ and AOB-t8. Importantly, a control compound BNC, in which the -OH group in azobenzene moiety is substituted with $-\mathrm{OCH}_{3}$, was synthesized to determine the binding site of ferric ion with AOB-t8. As expected, the FTIR spectra of BNC and BNC@ $\mathrm{Fe}^{3+}$ in Figure S4 also demonstrate insignificant change of the stretching vibration mode of $\mathrm{C}-\mathrm{N}$ at $1141 \mathrm{~cm}^{-1}$, due to there is no -OH group to bind with $\mathrm{Fe}^{3+}$ in BNC. Therefore, the binding site of AOB-t8 to $\mathrm{Fe}^{3+}$ is much likely to coordinate to the phenol $\mathrm{O}$ atom of $-\mathrm{OH}$ and $\mathrm{N}$ atom of azobenzene moiety. Based on the results of Job's plot and FTIR spectra, together with the possibility of geometric configuration, AOB-t8 is likely to form the dimer structure through the chelation of ferric ion with the azobenzene moiety[39]. In other word, the formation of 2:2 complex between $\mathrm{AOB}-\mathrm{t} 8$ and $\mathrm{Fe}^{3+}$ is the most likely binding mode, which can be further confirmed by MALDI-TOF-MS experimental result as shown in Figure S5. Clearly, the peak at $\mathrm{m} / \mathrm{z}=727.46$ is from the $\left[\mathrm{AOB}-\mathrm{t} 8+\mathrm{H}^{+}\right]$, and the peak at 780.88 is attributed to $\left[2 \mathrm{AOB}-\mathrm{t} 8+2 \mathrm{Fe}-4 \mathrm{H}^{+}\right]$. Schematically, the possible binding mode for AOB-t8 with ferric ion can be depicted as Scheme 2.

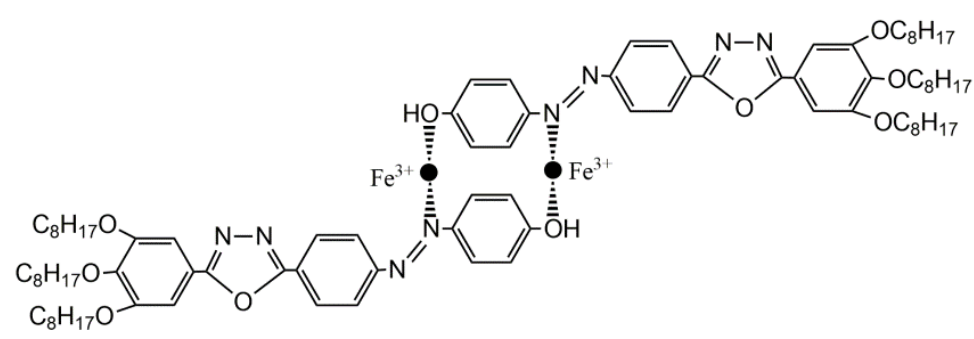

Scheme 2 The possible binding mode of $\mathrm{Fe}^{3+}$ with AOB-t8.

\subsection{Ion effect on photoisomerization}

The photoisomerization is an important photophysical characteristic of azobenzene derivatives. As is expected, the fluorescence of cis-AOB-t8 can also be quenched with the addition of $\mathrm{Fe}^{3+}$ (Figure S6), and the quenching mechanism for cis-AOB-t8 is believed to be the same as that for trans-AOB-t8. It was reported in our previous work that the isomerization from trans-AOB-t8 to cis-AOB-t8 can lead to a fluorescence emission enhancement[25]. Therefore, the fluorescence spectroscopy was employed to investigate 
the influence of ferric ion on the photoisomerization and geometrical configuration of AOB-t8. Apparently, the photoinduced isomerization of free trans-AOB-t8 $(10 \mu \mathrm{M})$ takes 55 min of UV light irradiation for the fluorescence intensity reaching the maximum, and it takes about $45 \mathrm{~min}$ in the case of trans-AOB-t8 with $\mathrm{Fe}^{3+}$ (Figure S7). Interestingly, the relationship of fluorescence with UV light irradiation time shown in Figure 6a clearly demonstrates the effect of ferric ion on the chemosensor AOB-t8. In the irradiation time from 0 to $35 \mathrm{~min}$, the emission intensity of trans-AOB-t8 with $\mathrm{Fe}^{3+}$ changes much faster than that of free trans-AOB-t8, and the rates of fluorescence evolution of trans-AOB-t8 and trans-AOB-t8-Fe(III) are 4.0 and 167.3 a.u./min, respectively. So, the chelation of $\mathrm{Fe}^{3+}$ accelerates the trans-to-cis photoisomerization of the azobenzene unit in AOB-t8. As is known, the geometrical configuration influences the optical properties and the molecular response to external stimuli, and the coplanar and rigid molecules usually demonstrate a faster response than the non-coplanar or flexible molecules. Therefore, it might be suggested that the chelation of $\mathrm{Fe}^{3+}$ leads to the generated complex much rigid and coplanar[40, 41], and thus the azobenzene moiety is easier to be isomerized from trans- to cis- conformation. This proposed mechanism is in good agreement with the hypsochromic shift of the absorption band shown in Figure 1b, indicating that the chelation of $\mathrm{Fe}^{3+}$ regulates the electronic state and molecular geometrical configuration of AOB-t8. Similar suggestion reported by Tamaoki also supports the point that the acceleration of isomerization of the azobenzene unit by $\mathrm{Fe}^{3+}$ ion is related to the strong perturbations of the electronic state and structure for both cis-AOB-t8-Fe(III) and trans-AOB-t8-Fe(III) [39].
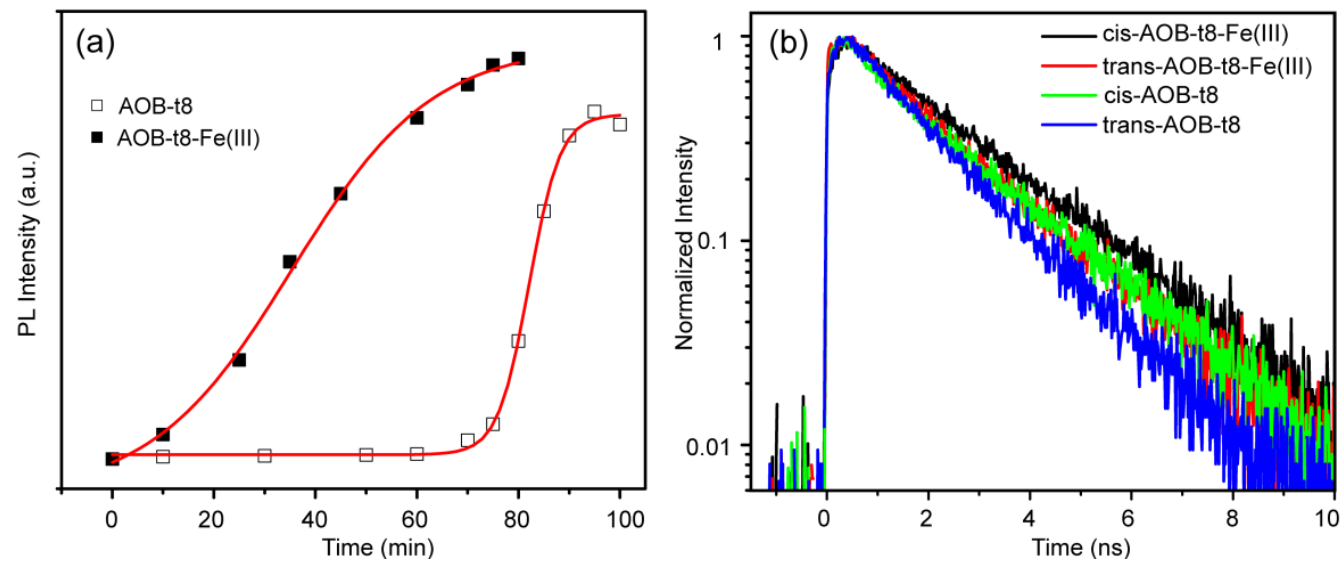
Figure 6 (a) Fluorescence change of AOB-t8 and AOB-t8-Fe(III) as a function of UV light irradiation time. (b) Fluorescence decay of trans- and cis-AOB-t8 as a function of time in the absence and presence of $\mathrm{Fe}^{3+}$. $[\mathrm{AOB}-\mathrm{t} 8]=10 \mu \mathrm{M}$, and $\left[\mathrm{Fe}^{3+}\right]=40 \times 10^{-5} \mathrm{M}$.

To verify the proposed chelation pattern and the involved mechanism of trans- and cis-AOB-t8 sensing to $\mathrm{Fe}^{3+}$, we carried out the fluorescence lifetime measurements for trans- and cis-AOB-t8 without and with the addition of $\mathrm{Fe}^{3+}$. As shown in Figure 6b, all of the fluorescence decay curves demonstrate the single exponential feature. Fitting the emission transients, the lifetimes of $1.54 \mathrm{~ns}$ for trans-AOB-t8 and $1.90 \mathrm{~ns}$ for cis-AOB-t8 can be obtained, respectively. As mentioned above, the binding of ferric ion leads to the absorption blue-shifting and the fluorescence quenching of AOB-t8, indicating the generated pathway to deactivate the perturbed excited state of host molecule. Logically, a shortening fluorescence lifetime should be expected with the chelation of metal ions. In stark contrast, the fluorescence lifetime of trans-AOB-t8 is increased to $1.85 \mathrm{~ns}$ and that of cis-AOB-t8 changes from 1.90 to $2.20 \mathrm{~ns}$ with the addition of $\mathrm{Fe}^{3+}$ ions. In general, the fluorescence intensity of a compound basically correlates with its extinction coefficient, excitation intensity, fluorescence quantum yield, fluorescence lifetime, energy transfer, etc. In other words, the decreased fluorescence intensity does not have to correlate with the decreasing of lifetime. It is a fact that the increase of molecular coplanarity and rigidity can impede the non-irradiative deactivation pathway and thus increase the fluorescence lifetime. Therefore, the inconsistency between the quenching emission intensity and increasing fluorescence lifetime suggests to a certain extent that the $\mathrm{Fe}^{3+}$ binding with AOB-t8 really regulates the molecular rigidity and coplanarity[42, 43], which is understandable from the proposed dimer binding mode.

\subsection{Sensing reversibility}

The sensor is always expected to demonstrate a reversible response in the practical applications. As one of the chelating ligands, citric acid is highly associable with many metal ions[44, 45]. It would not be surprised that the citric acid is able to associate with $\mathrm{Fe}^{3+}$ ions to form a very stable species $\mathrm{C}_{6} \mathrm{H}_{5} \mathrm{O}_{7} \mathrm{Fe}$ and displace the ligand AOB-t8 in AOB-t8-Fe(III). As expected, the fluorescence of AOB-t8-Fe(III) system clearly 
demonstrates a dramatic recovery with the addition of citric acid (Figure 7a and Figure S8). The reversibility of AOB-t8 sensor was tested by the alternate addition of ferric ion and citric acid into the solution. It is found from Figure $7 \mathrm{~b}$ that more than $74.4 \%$ quenched fluorescence by $\mathrm{Fe}^{3+}$ can be recovered in four test rounds. Additionally, the influence of citric acid on the change of fluorescence intensity at the test wavelength can be neglected in the experiments (Figure S9).
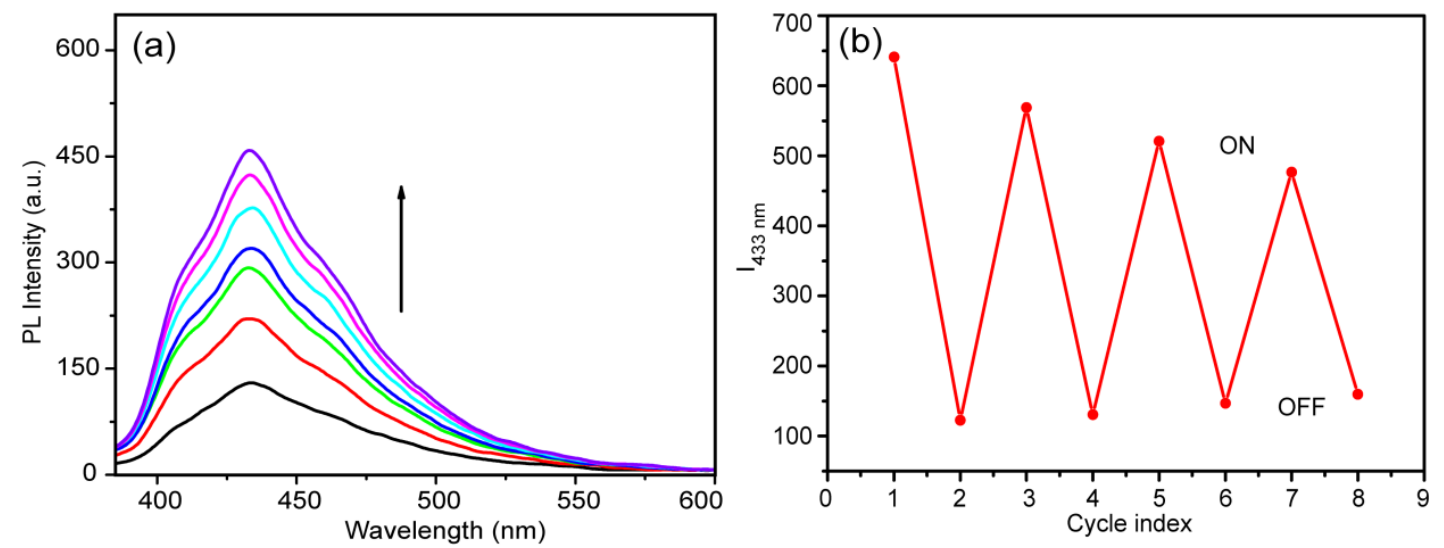

Figure 7 (a) Fluorescence spectra of trans-AOB-t8-Fe(III) with different concentrations of citric acid $(0,1,2,3,4,5,6) \mathrm{mM},[\mathrm{AOB}-\mathrm{t} 8]=10 \mu \mathrm{M}$, and $\left[\mathrm{Fe}^{3+}\right]=40 \times 10^{-5} \mathrm{M}$, (b) reversible switching cycles of fluorescence intensity by alternate addition of $\mathrm{Fe}^{3+}$ and citric acid in ethanol.

In summary, both trans- and cis-AOB-t8 demonstrate a specifically selective response to $\mathrm{Fe}^{3+}$ ions. The binding with ferric ion results in the absorption blue-shifting and fluorescence quenching of AOB-t8 due to the chelation and the intramolecular charge transfer. The dimerized chelation with $\mathrm{Fe}^{3+}$ ions is accomplished through the coordinating with phenol $\mathrm{O}$ atom of $\mathrm{OH}$ group in one molecule and $\mathrm{N}$ atom on the left side of azobenzene moiety in another AOB-t8. Consequently, the electronic state, molecular geometry and rigidity are perturbed, and the isomerization rate of trans-AOB-t8 to UV light irradiation is accelerated. The regulated molecular configuration is supported by the increased fluorescence lifetime of both trans- and cis- isomers. The reversible recognition and releasing of $\mathrm{Fe}^{3+}$ can be alternatively realized by adding citric acid, which is of significance in practical application for the present chemosensor.

\section{Conclusion}


We have investigated the ferric ion recognition characteristics of the chemosensor based on a photoresponsive azobenzene derivative by using various spectroscopies. The fluoroionophore AOB-t8 demonstrates a fluorescent turn-off sensor for ferric ion and exhibits good selectivity among 14 metal ions. The sensing of AOB-t8 to iron ion is accomplished through the dimerized chelation with $\mathrm{Fe}^{3+}$, resulting in the absorption blue-shifting and emission quenching. The dimer structure of AOB-t8-Fe(III) is formed through the coordinating of $\mathrm{Fe}^{3+}$ with $\mathrm{OH}$ group in one AOB-t8 and $\mathrm{N}$ atom on the left side of azobenzene moiety in another AOB-t8. The quenched fluorescence of both trans- and cis-AOB-t8 can be efficiently recovered with the addition of citric acid via a ligand displacement. Meanwhile, the chelation of $\mathrm{AOB}-\mathrm{t} 8$ with $\mathrm{Fe}^{3+}$ improves the response of photoisomerization and regulates the molecular rigidity or coplanarity.

\section{Acknowledgements}

This work was financially supported by the National Science Foundation Committee of China (project No. 21173068 and U1504510) and the Program for Innovative Research Team in University of Henan Province (No. 13IRTSTHN017).

\section{References and notes}

[1] H.S. Jung, P.S. Kwon, J.W. Lee, J.I. Kim, C.S. Hong, J.W. Kim, S. Yan, J.Y. Lee, J.H. Lee, T. Joo, J. Am. Chem. Soc. 131 (2009) 2008-2012.

[2] E.N. Kaya, F. Yuksel, G.A. Özpınar, M. Bulut, M. Durmuş, Sens. Actuat. B; Chem. 194 (2014) 377-388.

[3] H.N. Kim, M.H. Lee, H.J. Kim, J.S. Kim, J. Yoon, Chem. Soc. Rev. 37 (2008) 1465-1472.

[4] Y. Lu, S. Huang, Y. Liu, S. He, L. Zhao, X. Zeng, Org. Lett. 13 (2011) 5274-5277.

[5] M. Shellaiah, Y.H. Wu, H.C. Lin, Analyst. 138 (2013) 2931-2942.

[6] Y. Zhou, J. Zhang, H. Zhou, X. Hu, L. Zhang, M. Zhang, Spectrochim. Acta Part A Mol. Biomol.Spectrosc. 106 (2013) 68-72.

[7] J.S. Kim, M.G. Choi, K.C. Song, K.T. No, S. Ahn, S.-K. Chang, Org. Lett. 9 (2007) 1129-1132.

[8] L. Marbella, B. Serli - Mitasev, P. Basu, Angew. Chem. Int. Edit. 48 (2009) 3996-3998.

[9] R. Krämer, Angew. Chem. Int. Edit. 37 (1998) 772-773.

[10] M. Arduini, F. Mancin, P. Tecilla, U. Tonellato, Langmuir. 23 (2007) 8632-8636.

[11] T. Gunnlaugsson, J.P. Leonard, N.S. Murray, Org. Lett. 6 (2004) 1557-1560.

[12] X.Y. Chen, J. Shi, Y.M. Li, F.L. Wang, X. Wu, Q.X. Guo, L. Liu, Org. Lett. 11 (2009) 4426-4429.

[13] G.K. Tsikalas, P. Lazarou, E. Klontzas, S.A. Pergantis, I. Spanopoulos, P.N. Trikalitis, G.E. Froudakis, H.E. Katerinopoulos, RSC Advances. 4 (2014) 693-696.

[14] H. Wang, Y. Li, S. Xu, Y. Li, C. Zhou, X. Fei, L. Sun, C. Zhang, Y. Li, Q. Yang, Org. Biomol. Chem. 9 (2011) 2850-2855.

[15] J.Y. Kwon, Y.J. Jang, Y.J. Lee, K.M. Kim, M.S. Seo, W. Nam, J. Yoon, J. Am. Chem. Soc. 127 (2005) 10107-10111.

[16] J.L. Bricks, A. Kovalchuk, C. Trieflinger, M. Nofz, M. Büschel, A.I. Tolmachev, J. Daub, K. Rurack, J. Am. Chem. Soc. 127 (2005) 13522-13529.

[17] X. Chen, H. Hong, R. Han, D. Zhang, Y. Ye, Y.f. Zhao, J. Fluoresc. 22 (2012) 789-794. 
[18] P.K. Chung, S.R. Liu, H.F. Wang, S.P. Wu, J. Fluoresc. 23 (2013) 1139-1145.

[19] T. Michinobu, Y. Li, T. Hyakutake, Phys. Chem. Chem. Phys. 15 (2013) 2623-2631.

[20] Y. Zhou, Q. Huang, Q. Zhang, Y. Min, E. Wang, Spectrochim. Acta Part A Mol. Biomol.Spectrosc. 137 (2015) 33-38.

[21] Y. Norikane, N. Tamaoki, Org. Lett. 6 (2004) 2595-2598.

[22] S. Shinkai, T. Nakaji, Y. Nishida, T. Ogawa, O. Manabe, J. Am. Chem. Soc. 102 (1980) 5860-5865.

[23] Y. Wu, Y. Demachi, O. Tsutsumi, A. Kanazawa, T. Shiono, T. Ikeda, Macromolecules. 31 (1998) 349-354.

[24] T. Ikeda, J.i. Mamiya, Y. Yu, Angew. Chem. Int. Edit. 46 (2007) 506-528.

[25] X. Ran, H. Wang, L. Shi, J. Lou, B. Liu, M. Li, L. Guo, J. Mater. Chem. C. 2 (2014) 9866-9873.

[26] O. Yaroshchuk, Y. Reznikov, J. Mate. Chem. 22 (2012) 286-300.

[27] A.A. Beharry, G.A. Woolley, Chem. Soc. Rev. 40 (2011) 4422-4437.

[28] P. Gorostiza, E.Y. Isacoff, Science. 322 (2008) 395-399.

[29] X. Liang, H. Asanuma, M. Komiyama, J. Am. Chem. Soc. 124 (2002) 1877-1883.

[30] A.D. Wong, T.M. Güngör, E.R. Gillies, ACS Macro Lett. 3 (2014) 1191-1195.

[31] S. Samanta, A.A. Beharry, O. Sadovski, T.M. McCormick, A. Babalhavaeji, V. Tropepe, G.A. Woolley, J. Am. Chem. Soc. 135 (2013) 9777-9784.

[32] X. Ran, H. Wang, J. Lou, L. Shi, B. Liu, M. Li, L. Guo, Soft Mater. 12 (2014) 396-402.

[33] S. Hu, S. Zhang, C. Gao, C. Xu, Q. Gao, Spectrochim. Acta Part A Mol. Biomol.Spectrosc. 113 (2013) 325-331.

[34] L.J. Fan, Y. Zhang, C.B. Murphy, S.E. Angell, M.F. Parker, B.R. Flynn, W.E. Jones, Coordin. Chem. Rev. 253 (2009) 410-422.

[35] L. Wen feng, M. Heng chang, M. Yuan, Q. Chun xuan, Z. Zhon wei, Y. Zeng ming, C. Hai ying, RSC Advances. 5 (2015) 6869-6878.

[36] B.P. Joshi, J. Park, W.I. Lee, K.H. Lee, Talanta. 78 (2009) 903-909.

[37] X. Cao, W. Lin, L. He, Org. Lett. 13 (2011) 4716-4719.

[38] S. Bee, P. Agarwal, A. Gupta, P. Tandon, Spectrochimica Acta Part A Molecular \& Biomolecular Spectroscopy. 114C (2013) 236-255.

[39] Y. Oka, N. Tamaoki, Inorg. Chem. 49 (2010) 4765-4767.

[40] P.D. Beer, A.S. Rothin, Journal of the Chemical Society, Chemical Communications. (1988) 52-54.

[41] W.M. Bloch, A. Burgun, C.J. Coghlan, R. Lee, M.L. Coote, C.J. Doonan, C.J. Sumby, Nature Chem. 6 (2014) 906-912.

[42] K.K.W. Lo, J.S.Y. Lau, Inorg. Chem. 46 (2007) 700-709.

[43] V. Fernández Moreira, F.L. Thorp Greenwood, M.P. Coogan, Chem. Commun. 46 (2010) 186-202.

[44] Z. Lu, H. Chen, R. Robert, B.Y. Zhu, J. Deng, L. Wu, C.Y. Chung, C.P. Grey, Chem. Mater. 23 (2011) 2848-2859.

[45] F. Tian, E.A. Decker, J.M. Goddard, J. Agr. Food Chem. 61 (2013) 12397-12404. 


\section{Table of Contents}
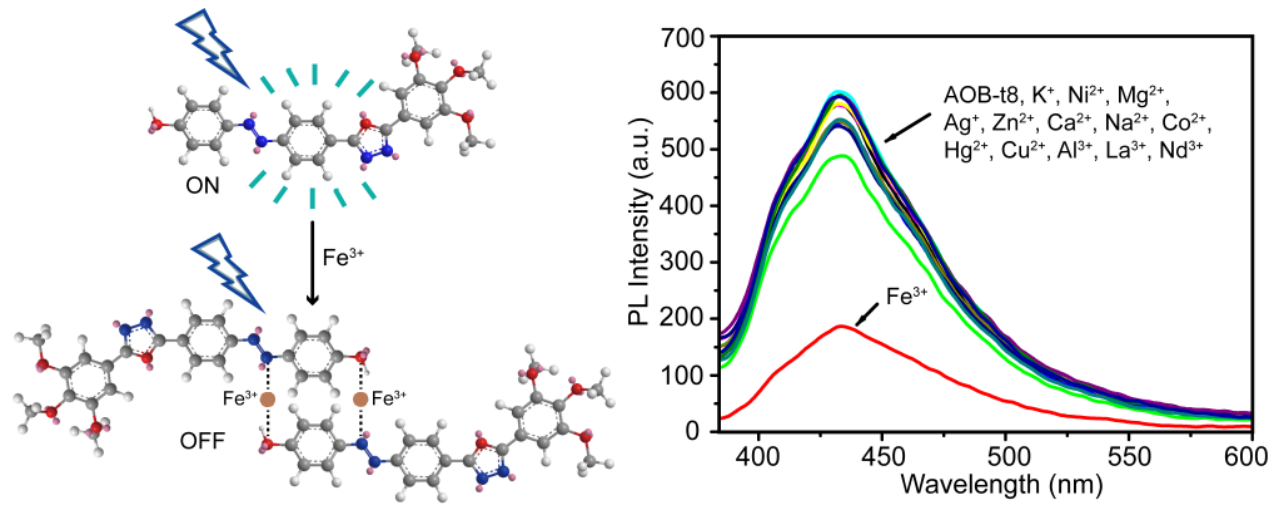

Fluorescent sensor of AOB-t8 shows high selectivity for ferric ion. (In order to convenient show the structural formula, the 3,4,5-octanoxy group of AOB-t8 was replaced by three methoxy groups. ) 